Bucknell University

Bucknell Digital Commons

\title{
Identification of speeded and slowed familiar melodies by younger, middle-aged, and older musicians and nonmusicians
}

\author{
M.W. Andrews \\ W.J. Dowling \\ J.C. Bartlett \\ Andrea Halpern \\ Bucknell University, ahalpern@bucknell.edu
}

Follow this and additional works at: https://digitalcommons.bucknell.edu/fac_journ

Part of the Neuroscience and Neurobiology Commons

\section{Recommended Citation \\ Andrews, M.W.; Dowling, W.J.; Bartlett, J.C.; and Halpern, Andrea. "Identification of speeded and slowed familiar melodies by younger, middle-aged, and older musicians and nonmusicians." Psychology and Aging (1998) : 462-471.}

This Article is brought to you for free and open access by the Faculty Scholarship at Bucknell Digital Commons. It has been accepted for inclusion in Faculty Journal Articles by an authorized administrator of Bucknell Digital Commons. For more information, please contact dcadmin@bucknell.edu. 


\title{
Identification of Speeded and Slowed Familiar Melodies by Younger, Middle-Aged, and Older Musicians and Nonmusicians
}

\author{
Melinda W. Andrews, W. Jay Dowling, \\ and James C. Bartlett \\ University of Texas at Dallas
}

\author{
Andrea R. Halpern \\ Bucknell University
}

\begin{abstract}
There is a range of tempos within which listeners can identify familiar tunes (around 0.8 to 6.0 notes $/ \mathrm{s}$ ). Faster and slower tunes are difficult to identify. The authors assessed fast and slow melodyidentification thresholds for 80 listeners ages 17-79 years with expertise varying from musically untrained to professional. On fast-to-slow (FS) trials the tune started at a very fast tempo and slowed until the listener identified it. Slow-to-fast (SF) trials started slow and accelerated. Tunes either retained their natural rhythms or were stylized isochronous versions. Increased expertise led to better performance for both FS and SF thresholds $(r=.45)$. Performance declined uniformly across the 62-year age range in the FS condition $(r=.27)$. SF performance was unaffected by age. Although early encoding processes may slow with age, expertise has a greater effect. Musical expertise involves perceptual learning with melodies at a wide range of tempos.
\end{abstract}

The slowing of certain cognitive processes with age is a welldocumented phenomenon (Salthouse, 1996a, 1996b). Tasks that show this effect include verbal and visual memory, judgment, mental arithmetic, and tests of spatial ability. Research in this area has generally been concerned with how fast people of different ages could accomplish information-processing tasks. With increasing age from around 20 years there is a gradual, steady decline in the speed of performance of speeded tasks. However, researchers have seldom asked how slowly people could perform tasks that require slow performance to be effective. For example, certain pieces of music are more effective when slow, though performing a piece extremely slowly, yet coherently, can be very difficult. Elisabeth Grümmer and Wilhelm Furtwängler's 1954 performance of the aria "Und ob die Wolke sie verhulle (And Even When the Clouds Hide It)" from Weber's Der Freischütz (The Freeshooter) owes much of its unique beauty to the especially slow tempo that they achieve. And there are situations where it is necessary to speak at an unusually slow tempo in order to be understood, as when talking with someone with a severe hearing loss or someone with only a beginning grasp of the language or when lecturing to a class with only a beginning grasp of the subject matter.

Several theoretical accounts have been developed to explain the slowing of cognitive processing with age. The simplest hy-

Melinda W. Andrews, W. Jay Dowling, and James C. Bartlett, Programs in Psychology and Cognitive Science, University of Texas at Dallas; Andrea R. Halpern, Department of Psychology, Bucknell University.

This research was supported by National Institute on Aging Grant 1-R01-AG09965. We thank Laura Straiter for assistance in data collection, Janie Jury for assistance in preparing the figure, and Bruno Repp and Richard Warren for helpful and thought-provoking comments.

Correspondence concerning this article should be addressed to W. Jay Dowling, Programs in Psychology and Cognitive Science, University of Texas at Dallas, Richardson, Texas 75083-0688. Electronic mail may be sent to jdowling@utdallas.edu. pothesis concerning generalized slowing involves an analogy to the central-processing clock on a computer. If the clock speed is slowed, then all the operations of the computer will be slowed accordingly, with far-reaching ramifications. A general slowing of information-processing speed in conjunction with the steady loss of information from memory could account for observed memory deficits, for example, as Charness (1985) has pointed out.

Two major alternatives to the clock-speed-slowing hypothesis are the increased-overhead hypothesis of Cerella (1990) and the information-loss model (Myerson, Hale, Wagstaff, Poon, \& Smith, 1990). Cerella has suggested that with increasing age an increasing number of links in the information-processing chain become damaged. This results both in the loss of information and in increased processing time, because previously used pathways must be replaced by more circuitous routes in the information-processing sequence (Cerella, 1990). Such a model accounts for deficits in both time and accuracy of information processing as more and more links are damaged with age. The closely related information-loss model of Myerson et al. (1990) attributes slowing to information loss during processing. This model assumes an increase in information loss with age and that the time required for each step of processing depends on the amount of information available at that step. Thus increased loss of information leads to a decrease in processing speed.

A fourth theoretical possibility suggested by Salthouse's (1996a, 1996b) reviews of the literature is that initial stimulus encoding processes become slower with age. This possibility is consistent with evidence that older listeners often show the most slowing with novel stimuli, and that older experts in a domain can sometimes use their domain-specific knowledge to compensate for slower encoding processes. Salthouse (1984) found such a pattern for expert typists, who were able to compensate for slowed response times by looking farther ahead in the text. And in the musical domain, Krampe and Ericsson (1996) showed that with older pianists deliberate practice serves to 
maintain musical performance skills at a high level of proficiency. We wished to test the generality of this pattern with the melody-identification task with which we could study listeners with very little formal musical training as well as experts. This task also provided a domain in which performance with extremely slow perceptual patterns is as important as performance with fast patterns.

To investigate these theories of cognitive slowing with age we used a task developed by Warren, Gardner, Brubaker, and Bashford (1989, 1991), who measured identification thresholds for speeded and slowed melodies. It seems intuitively obvious that a melody could go too fast to be recognized or identified, and in fact familiar melodies become difficult to identify at tempos faster than about 6 notes/s. Warren et al. (1989, 1991) also demonstrated the less obvious phenomenon that a melody can go too slow to be identified. A phrase of a familiar melody played very slowly (around 0.25 note/s) is all but unrecognizable. It is only when the tempo is increased to about 0.8 note/s that listeners can identify the tune. In Warren et al.'s procedure there are fast-to-slow (FS) trials that measure the fastest tempo at which listeners can identify a melody, and slowto-fast (SF) trials that measure the slowest tempo at which identification occurs. These two thresholds describe a tempo window within which listeners are able to identify a melody, roughly between 0.8 and 6 note $/ \mathrm{s}$.

Our first goal in the present experiment was to use Warren et al.'s $(1989,1991)$ procedure to examine age differences in listeners' ability to identify melodies at very fast tempos and at very slow tempos. Our second goal was to find out whether music training might moderate any age differences we obtained. Listeners ranged in age from 17 to 79 years, and varied widely in musical expertise. We wanted to evaluate the theories of cognitive slowing in aging noted previously.

The predictions of the four theories concerning the effects of aging on the tempo window for melody identification are diagrammed in Figure 1. For each theory an open bar represents the hypothetical performance of younger listeners at fast and slow tempos; a filled bar represents the thresholds of older listeners. We shall first present the predictions of the theories, followed by the detailed bases for those predictions. All of the theories predict slower performance with age at the fast end of the continuum. The increased-overhead and information-loss models predict difficulty handling slowed-processing tasks as well as fast-processing tasks, with faster identification thresholds for slow melodies. With the increased-overhead model this occurs because lengthening processing times allow information concerning later notes to disappear before processing of earlier notes is finished. Similarly, in the information-loss model earlier notes in the melody risk being lost by the time later notes arrive. The initial-encoding-difficulty theory, while predicting a slowing of the fast threshold, does not predict a speeding up of the slow threshold.

The slowed-clock model, in contrast, makes a unique and straightforward prediction about how slow processing can go when slowness is required. If slowing with age is due simply to the general slowing of the pace of information processing, then all of the person's information processing will be slowed accordingly and this will be reflected both in speeded tasks (where performance will be slowed) and slowed tasks (where
TEMPO

SLOW

FAST

THEORY

SLOWED CLOCK

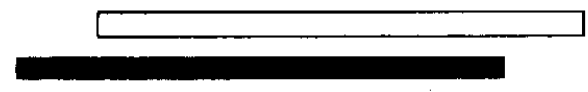

INCREASED OVERHEAD

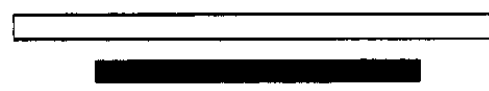

INFORMATION LOSS

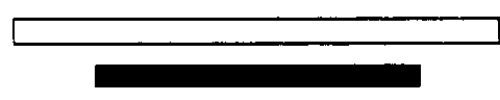

INITIAL ENCODING

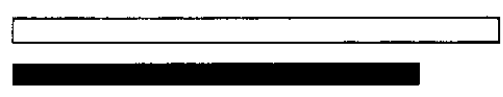

Figure 1. Predictions made by four theoretical models concerning fast and slow thresholds for tune identification by younger (open bars) and older (filled bars) listeners.

performance will also be slowed). That is, according to the slowed-clock model older listeners should show a deficit with speeded tasks, but should perform better than younger listeners with slow tasks.

Thus we have a task that can distinguish in part among these theories. However, melody identification at very rapid and very slow tempos is a complex task involving multiple components that are different at the two ends of the tempo continuum. A more precise characterization of our predictions depends upon a more detailed task analysis of the Warren et al. (1989) paradigm.

\section{Task Components}

Melody identification requires encoding of stimulus elements, maintaining serial order relationships in memory, parsing the ongoing stream of stimulus elements into meaningful groups (gestalts), and holding stimuli in working memory long enough for the pattern to be identified. The melody-identification task poses different problems for the listener at very fast tempos than it does at very slow tempos.

\section{Fast-Melody Identification}

For fast melodies, melody identification requires rapid encoding of stimulus elements. At the upper limit of the melodyidentification window, notes are arriving at rates of 6 or 8 per second. This is on the upper edge of the rate of syllable presentation in speech (Warren et al., 1991), and it is at rates of 6 to 8 notes/s that errors appear in pitch encoding. For example, at 3 notes/s the pitches of target notes in a stream are encoded accurately, whereas the introduction of distractor notes boosting the presentation rate to 6 notes/s leads to assimilation errors in the encoding of the pitches of quarter steps (that lie between the standard semitones of the musical scale; Dowling, 1992). 
As indicated previously, beyond about 10 items/s both speech sounds and musical tones are no longer resolved as sequential items, but blend together into a perceptual whole qualitatively different from the original word or melody (Warren \& Bashford, 1993). Thus in the tempo range of 6-10 notes/s the encoding processes for individual items in a sequence are being stretched to the limit.

A second possible problem encountered in fast melody identification is that of encoding the serial order relationships among the notes, an essential property of the melody. This may be a particular problem for older listeners, as Trainor and Trehub (1989) found. Trainor and Trehub investigated whether lowlevel sensory processing in audition, as instantiated by the streaming effect of rapid sequences of tones in different pitch ranges, would be affected by aging. This was not the case. Rather, age appeared to be associated with a problem in keeping straight the order of the pitches in a sequence. Thus aging brings about problems in the early encoding of the stimulus sequence, rather than its initial parsing into streams.

Third, with the rapidly cycling melodic phrase, identification depends upon identifying where in the tone sequence the melody begins; that is, parsing the stream into recurring instances of the repeating phrase. This should be especially difficult with isochronous melodies in which rhythmic cues to the start and finish of the phrase have been eliminated.

At the fast end of the tempo continuum, working memory capacity in terms of time span should not be a problem, because the whole phrase fits easily into the 4 or $5 \mathrm{~s}$ typically occupied by naturally occurring melodic phrases (Dowling \& Harwood, 1986). Working memory capacity in terms of number of elements should not be a problem either, because at a fast tempo notes initially encoded for temporal order are more likely to be grouped into phrases ("chunked") for later processing. To the extent that phrases are difficult to form, expertise plays a role in facilitating grouping. As Warren et al. (1991) have suggested, at fast tempos "special organizational rules governing the perception of tonal sequences" (p. 282) may come into play. The perception of such domain-specific pattern organization should be facilitated by musical training. It may be that moderately experienced listeners are able to encode the melodies they hear in terms of a quasi-notational representation, as Dowling (1986) had suggested. But whether the representation depends on musical notation or not, more experienced listeners should be able to use a more sophisticated representation than the less experienced in solving the melody-identification task.

\section{Slow-Melody Identification}

With slow melodies listeners should have ample time to encode stimulus elements, and so encoding should pose no problem. Keeping the order of elements straight may be a problem, but with slow melodies may be more of a memory problem than a perceptual one. The temporal capacity of the workingmemory buffer may easily be exceeded, and so it is conceivable that a listener might be able to remember that a particular pitch had been presented, but not to remember the temporal relationships among the pitches. In general, a melody stretched out over $30 \mathrm{~s}$ in time is difficult to represent in working memory, because
$30 \mathrm{~s}$ far exceeds the usual melodic phrase length of under $5 \mathrm{~s}$ (Dowling \& Harwood, 1986).

Warren et al. (1989) observed that at slow tempos "melodic cohesion was lost, and each note was heard as an independent sound" (p. 347). They reported that at least some listeners adopted a strategy of "remembering the intervals from one tone to the next as they occurred at the slow rates, and then "playing back' from memory at a faster rate," and added that "reconstruction of the melodies from these successive tones was difficult without formal training in music." They suggested that those "with formal training used musical notations as an aid, and could reconstruct a melody readily, much as a word can be identified by literate listeners when they hear it spelled' (Warren et al., 1989, p. 347). Following such a strategy clearly puts a load on working memory.

The parsing problem is at least as difficult for slow melodies as for fast melodies, but takes a somewhat different form. If only a certain number of separate notes (for example, seven) can be held in working memory at a time, then ensuring that those are the first seven notes of the melody facilitates the task immensely. The listener's ensuring that a certain set of notes forms a coherent phrase, rather than being the end of one phrase and the beginning of another, makes identification much easier. Thus being able to identify the starting point of the repeating melody improves performance.

In summary, three information-processing components (maintaining serial order, parsing, and memory load) would appear to affect both fast and slow thresholds, whereas the fourth component, stimulus encoding, would appear to affect only fast performance.

\section{Component Tasks and Theoretical Models}

As noted previously, the slowed-clock model predicts declining performance with age for fast-melody identification, but better performance (that is, slower identification thresholds) for slow melodies. In three components of the identification taskmaintaining serial order, parsing, and memory load-both the increased-overhead and information-loss models would predict worse performance with age for both fast and slow identification, and thus slower thresholds for fast melodies and faster thresholds for slow melodies. This is because those components involve memory operations that would be slowed and made more fallible by either the loss of processing links or of information. Clearly, with fast melodies disruption of the rapid information processing required delays identification. Disruption should occur with slow melodies as well. Part of the listener's problem in creating a viable gestalt with a slow stimulus is in holding the successive notes of the melody in memory while waiting for the next note to arrive. To the extent that the memory system is made more fallible by the disruption of processing links or loss of information, those operations are made more difficult and prone to error, and the identification of a given melody is delayed. With the present method of limits, the delay means that the melody has speeded up and the measured threshold is faster. Keeping track of serial order and parsing the melodic phrase put similar loads on memory, as indicated previously. Thus to the extent that the processing of slow melodies puts more load 
on memory, these models predict greater age deficits with slow melodies than with those of moderate speed.

Thus in contrast to the slowed-clock model, the increasedoverhead and information-loss models predict a shift toward worse performance at both ends of the spectrum: Fast thresholds should become slower, and slow thresholds faster, with age.

Note from the analysis of task components that a deficit in encoding processes leads to a unique pattern of predictions for FS and SF performance; namely, that problems with encoding lead to worse performance on FS trials but should not affect SF performance. To the extent that fast thresholds decline with increasing age, with no change in the slow thresholds, then that is probably not the effect of a generalized slowing mechanism operating on both perception and memory such as those of the slowed-clock, increased-overhead, and information-loss models. Rather, such results implicate a slowing mechanism involved specifically with stimulus encoding, and would thus converge with a wide variety of results assembled by Salthouse (1996b).

\section{Aging and Expertise}

Aging and expertise should have different effects on the various task components just described. In general, aging should be associated with slowed information processing as outlined previously. Expertise should improve performance in ways associated with more efficient encoding processes that take advantage of stimulus redundancies reflected in perceptual learning of experts, improved parsing and order-maintaining processes that derive from increased familiarity with the materials, and more efficient grouping of stimulus elements into larger meaningful groups through chunking (thereby reducing memory load). The present study seeks to determine whether the effects of expertise can offset those of aging.

\section{Design of Experiment}

In order to investigate the effects of aging and expertise on fast and slow thresholds for melody identification we designed an experiment in which separate blocks of six trials each tested each of six different conditions. Listeners in three different age ranges $(17-30,31-59$, and $60-79$ years) and three different levels of expertise from inexperienced to professional served in all six conditions. On each trial a familiar melody would be repeated until the listener identified it. The melody began in the middle. In half the conditions the melody started very slow and gradually sped up (the SF condition), and in the other half the melody began very fast and slowed down (the FS condition). The melodies were presented either with their familiar rhythmic patterns (the rhythmic condition: $R$ ) or with notes all of equal length (the isochronous condition: $\mathrm{I}$ ). We introduced the latter condition because in melodies where the shortest notes were one eighth as long as the longest notes, the short notes would become vanishingly small at the fastest tempos. We expected that isochronous presentation would aid performance in the FS condition.

Four of the six conditions were arranged in a $2 \times 2$ design in which starting speed (SF vs. FS) was crossed with rhythm ( $R$ vs. I). To those four conditions we added a variant of the FS condition in which a temporal gap was introduced between the repetitions of the melodic phrase. This was intended to aid parsing. We thought that if parsing posed a particular problem for the oldest listeners (cf. Trainor \& Trehub, 1989), then we should observe an improvement in performance between the regular FS-R and FS-I conditions and those with the gap (FSG$R$ and FSG-I) for that group. We did not introduce a gap in the SF conditions, principally because to be noticeably longer than the longest delays between the onsets of notes at $4 \mathrm{~s} /$ beat, such gaps would have to be extremely long. (Consider that at such a tempo $8 \mathrm{~s}$ separates a half note [ 2 beats] from its successor. If the phrase ends in a half note, then that [ $8 \mathrm{~s}$ ] delay must be added to the delay for the gap [more than $8 \mathrm{~s}$ ]. Such a procedure would have sorely tried the listeners' patience, as well as lengthened the session considerably.)

Method

\section{Listeners}

Eighty listeners served in individual sessions. Listeners were either paid $\$ 10.00$ for the approximately 40 -min session, or, if they were students, received class credit. Listeners were grouped by age: younger (age 17-30 years, $N=24, M=24.0$ years, 4 men and 20 women), middle ( $31-59$ years, $N=27, M=39.2$ years, 5 men and 22 women), and older $(60-79$ years, $N=29, M=67.1$ years, 15 men and 14 women ). Listeners were grouped by musical experience on the basis of a brief questionnaire on which they were asked to specify ages at which they had had particular musical training and performance experience. The groups classified by musical experience were: inexperienced (up to 2 years of formal musical training, $N=31$ ), moderately experienced (between 2 and 10 years of formal musical training, but not professional, $N=25$ ), and professional musicians (who had been paid to work as a musician during some part of their life, and who generally had much more than 10 years of experience, $N=24$ ). Inexperienced listeners averaged 0.3 years of music lessons; 19 listeners in that group had had no lessons, eight had had about 6 months of lessons, and four had had about 1 year; the median for that group was 0.0 years. Moderately experienced listeners averaged 5.2 years (typically taken between the ages of 8 and 20). Professionals had generally been practicing music actively and continuously from the age of 6 or so. Listeners were recruited by advertisements in the community and by personal contacts, as well as from the psychology student subject pool. There were 8 to 13 listeners in each of the nine cells of the 3 ages $\times 3$ experience levels design. The additional listeners (beyond 8 ) in the inexperienced category were typically spouses of professionals needed in those groups.

Older listeners received a brief audiometric screening involving measurement of pure-tone thresholds for four points between 250 and 8000 Hz. All participants had age-normal hearing in at least one ear. The experimenter adjusted the intensity of the stimuli so that the listener heard them clearly at a comfortable level.

All listeners completed a vocabulary test modeled on the second half of the vocabulary test from the Wechsler Adult Intelligence ScaleRevised (WAIS-R; Wechsler, 1981). The vocabulary test provided a measure of cognitive performance dissimilar to the tasks under investigation. A 3 ages $\times 3$ experience levels analysis of variance (ANOVA) on vocabulary performance disclosed only a main effect of age, with the youngest and oldest participants performing about equally well ( $63 \%$ and $61 \%$ correct, respectively), and the middle-aged group performing somewhat better $(74 \%), F(2,71)=4.11, p<.05, R^{2}=.097$. Thus age-related declines in the music tasks could not be attributed to general cognitive impairment. 


\section{Stimuli}

The stimuli were produced at comfortable levels by a synthesizer, using its acoustic piano voice and its own amplifier and speaker system. The synthesizer was under the control (by means of MIDI interface) of an IBM-compatible computer running Cakewalk software (Hendershott, 1990 ).

We used nine melodies that in our previous work had proved to be familiar to listeners of all ages in the experiment. Each stimulus consisted of the first two phrases (four measures) of the familiar song, repeated over and over. On each trial the stimulus pattern began at a point arbitrarily chosen in the middle of the song. There were two types of trial: fast-slow (FS), in which the stimulus pattern began fast ( 32 beats/s) and gradually slowed down, and slow-fast (SF), in which the pattern began slow ( .25 beats/s) and gradually speeded up. It was important that the starting points of FS and SF trials be well beyond the thresholds of melody identification found by Warren et al. (1991).

Each melody in each condition was tested with both an FS and an SF trial. On FS trials the rate of presentation was halved every six repetitions, with three extra repetitions added at the start to give the listener time to orient to the stimulus. On SF trials the rate doubled on the first repetition, and then doubled over the next two repetitions, and then doubled over the next three repetitions, etc., until it was doubling every six repetitions, from which point its rate of change remained constant. Changes in tempo from beat to beat were linearly interpolated between the points just described, beginning at the start of the trial. Each trial was terminated when the listener indicated by pressing a key on the synthesizer keyboard that he or she recognized the melody.

There were two rhythmic conditions under which the melodies were presented: isochronous (I) and rhythmic ( $R$ ). In the I condition the rhythms of the melodies were stylized so that they were presented in notes of equal length. In the $\mathbf{R}$ condition the melodies retained their normal rhythms. Of the nine melodies used in the experiment, three appeared in both the I and R conditions ("Happy Birthday," "London Bridge," and "Yankee Doodle" ), three appeared only in the I condition ("Frere Jacques," "Twinkle, Twinkle, Little Star," and "Old Macdonald"), and three appeared only in the $\mathbf{R}$ condition ("Swanee River," "'Rock of Ages," and "Rudolph, the Red-Nosed Reindeer"). The isochronous-only melodies were chosen because of their preponderance of equal note values, and the rhythmic-only melodies were chosen because of their characteristic rhythmic patterns, including variety of note lengths and identifiable rhythms. All the rhythmic melodies were easily recognizable at moderate tempos in isochronous versions (Dowling, 1973).

We included an additional version of the FS trial type, called FastSlow-Gapped (FSG). On FSG trials successive repetitions of the target melody were separated by a temporal gap of two beats in order to mark the beginning of the melodic pattern. Listeners completed six isochronous and six rhythmic trials with the FSG trials, just as with the FS trials.

\section{Procedure}

Listeners served in individual sessions at the University of Texas Dallas laboratory, seated at the synthesizer keyboard next to the experimenter, who operated the computer with the screen hidden from the listener. At the start of the session the listener heard each of the melodies at a moderate rate, identified by name. Listeners professed to be at least moderately familiar with the tunes. Each listener performed the conditions of the experiment in an individually determined random order, different from all other listeners. During the experiment listeners had in front of them a list of melody titles used in the current condition. The list included the title "Mary Had a Little Lamb," a tune not used in the experiment, to counteract the listener's possible strategy of eliminating the titles one by one throughout the block. Each listener completed six blocks of six trials each, one block for each condition: FS-I, FS-R,
SF-I, SF-R, FSG-I, and FSG-R, in random order. As noted previously, on each trial the melody was repeated at a constantly changing tempo, and the listener pressed a key on the keyboard when the melody became identifiable. The experimenter then stopped the stimulus presentation, and the computer stored the time of the listener's keyboard response. The listener then told the experimenter the name of the melody, and the experimenter noted the name and proceeded to the next trial. On rare occasions on which the listener appeared unsure of the response, or had pressed the key accidentally, the experimenter continued the trial and took another reading of the tempo threshold when the listener was sure of the identification.

\section{Data Analysis}

The listeners' responses were scored for correctness, and median tempo thresholds were analyzed both in terms of all responses and of correct responses only. Those two sets of analyses were in general agreement. The analyses for correct trials are reported here. Error data are presented later.

We calculated median tempo thresholds for each listener for each block of six trials. We used the median to minimize the effect of extreme outliers in the distributions-always a possibility with this type of response-time data. Median tempo thresholds were expressed as the tempo at which identification occurred. Tempo was calculated in terms of both milliseconds per beat and milliseconds per note, and separate analyses were carried out on the two sets of data. The results of the two sets of analyses were in qualitative agreement. Milliseconds per note is clearly the fairer way to compare the I and R conditions, because it refers to the amount of information transmitted over time. Therefore that is the measure that is reported here.

We also compared thresholds for the three particular tunes that were used in both the I and R conditions. That analysis is not reported because it does not add anything qualitatively different to the results of the overall analyses. It shows principally that listeners at different ages and experience levels find different tunes somewhat easier than others; that is, the analysis shows interactions of the listener variables with particular tunes and little else. Therefore tunes as a variable is not considered further.

\section{Results}

\section{Correlations With Age and Experience}

The most direct test of the effects of age and experience on FS and SF performance was to assess the correlations between median thresholds (for FS and SF trials separately, collapsed across I and $\mathbf{R}$ conditions), and age and experience. Here age was coded in years and experience coded on a 4-point scale, breaking the middle category of the three-way coding into two subcategories. The four categories were: less than 2 years musical experience, 2-5 years of experience, more than 5 years experience but not professional, and professional. We used this four-way encoding of experience because, unlike years of musical experience tout court, for example, it is not itself correlated with age, but nevertheless provides more information than the three-way categorization. Note that more information is preserved in the coding of age than of experience, and so the tests involving age are the more powerful tests. We revert to a threeway coding of experience for the ANOVAs reported later, however, because the four-way coding would lead to too small $n$ s in the cells. The results are shown in Table 1. There was a significant linear relationship between FS thresholds and age across the 62 -year range of the study, explaining about $7 \%$ of 
Table 1

Correlations Between Age and Experience and Recognition

Thresholds in Milliseconds/Note for

the FS and SF Conditions

\begin{tabular}{lcc}
\hline Factor & Age & Experience \\
\hline FS & $.27^{*}$ & $-.46^{* *}$ \\
SF & -.05 & $.45^{* *}$ \\
Experience & -.05 & \\
\hline
\end{tabular}

Note. $\quad$ FS $=$ fast-to-slow; $\mathrm{SF}=$ slow-to-fast.

$* p<.01 . \quad{ }^{* *} p<.001$

the variation in thresholds. The positive correlation indicates slower thresholds (in milliseconds per note) at greater ages. This effect was spread evenly across the age range of the study, as is seen in the ANOVAs. There was no significant relationship between age and SF performance or between age and experience. Expertise showed a stronger relationship to both FS and SF thresholds than age, in each case explaining about $20 \%$ of the variance. Here the negative correlation for FS trials indicates faster thresholds with greater expertise, and the positive correlation for SF trials indicates slower thresholds, that is, better performance in both cases.

If there were a general slowing of cognitive processing with age in accord with the slowed-clock hypothesis, then there should have been positive correlations between age and both SF and FS performance, a result that did not obtain. The overhead and information-loss models, on the other hand, predict a positive correlation with age on FS trials and a negative correlation on SF trials (that is, that older listeners would do worse in both conditions). That result did not obtain, either.

\section{Overall ANOVA}

In our ANOVAs we first analyzed all the median tempo thresholds (in milliseconds per note) obtained from the SF and FS conditions combined. Since there was no SF condition with a temporal gap between repetitions of the melody, we did not include the FSG condition in this analysis, but only the SF and FS conditions; the FSG condition is compared to the FS condition in a subsequent analysis. Second, because for most purposes averaging the results of SF and FS trials does not make sense, we subsequently performed separate analyses for the SF and FS conditions, and also compared the FS and FSG conditions. We report the analyses of just the correct trials, and note those cases in which the analyses of all the trials differ from those.

The overall ANOVA was on the design: $3 \times 3 \times 2 \times 2$ (Ages $\times$ Experience Levels $\times$ Speeds [FS vs. SF] $\times$ Rhythmic Conditions [R vs. I]). The effect of speed was of course significant, $F(1,71)=1,286.07, p<.001, M S E=343,540.3, R^{2}$ $=.825$, with FS thresholds faster than SF thresholds $(183 \mathrm{~ms} /$ note vs. $2,563 \mathrm{~ms} /$ note). In effect those thresholds bracket the region within which a familiar melody is recognizableroughly between 4 notes $/ \mathrm{s}$ and 0.4 notes $/ \mathrm{s}$. The effect of age was not significant so, as indicated in the correlational analysis, there was no overall slowing effect on identification thresholds with age. The effects of rhythmic condition and experience were
Table 2

Recognition Thresholds and Standard Deviations in Milliseconds/Note for SF, FS, and FSG Conditions for Listeners at Three Experience Levels

\begin{tabular}{crrrr}
\hline & \multicolumn{4}{c}{ Experience } \\
\cline { 2 - 4 } Condition & Low & Mid & Pro & $M$ \\
\hline SF & & & & \\
$M$ & 2,206 & 2,574 & 2,908 & 2,563 \\
$S D$ & 697 & 620 & 539 & \\
FS & & & & \\
$M$ & 204 & 183 & 162 & 183 \\
$S D$ & 54 & 50 & 56 & \\
FSG & & & & \\
$M$ & 198 & 183 & 145 & 175 \\
$S D$ & 55 & 54 & 46 & \\
\hline
\end{tabular}

Note. $\quad \mathrm{SF}=$ slow-to-fast $; \mathrm{FS}=$ fast-to-slow; FSG $=$ fast-to-slow, gapped; Pro $=$ professional .

significant, but are not meaningful when averaged across FS and SF conditions, because better performance lies in opposite directions in those two conditions. The interaction of experience with speed was significant, $F(2,71)=10.81, p<.001, M S E$ $=343,540.3, R^{2}=.014$, with more experienced listeners performing better for both the FS and SF thresholds, as found in the correlational analysis and as shown in Table 2. At the slow end each level of expertise is worth about a $15 \%$ increment in the threshold, whereas at the fast end it is worth about $11 \%$. The Rhythmic Condition $\times$ Speed interaction was significant, $F(1,71)=100.84, p<.001, M S E=86,937.1, R^{2}=.016$, with an advantage for isochronous presentation on FS trials, and for rhythmic presentation on SF trials, as shown in Table 3. That is, listeners achieved slower thresholds with rhythmic (vs. isochronous) slow melodies, and faster thresholds with isochronous (vs. rhythmic) fast melodies. No other effects were significant, and in particular there were no main effects or interactions involving age.

Table 3

Recognition Thresholds and Standard Deviations in Milliseconds/Note for FS and SF Conditions for Isochronous (I) and Rhythmic (R) Presentation

\begin{tabular}{crrr}
\hline & \multicolumn{2}{c}{ Rhythmic condition } & \\
\cline { 2 - 3 } Condition & \multicolumn{1}{c}{$\mathrm{I}$} & $\mathrm{R}$ & $M$ \\
\hline $\mathrm{SF}$ & & & \\
$M$ & 2,190 & 2,889 & 2,540 \\
$S D$ & 652 & 609 & \\
FS & 174 & 198 & 186 \\
$M$ & 53 & 54 & \\
$S D$ & & & \\
FSG & 166 & 191 & 179 \\
$M$ & 49 & 55 & \\
$S D$ & &
\end{tabular}

Note. $\mathrm{FS}=$ fast-to-slow; $\mathrm{SF}=$ slow-to-fast; FSG $=$ fast-to-slow, gapped. 
Slow-Fast

Next we separated the analyses of the SF and FS conditions. We analyzed the SF thresholds with a $3 \times 3 \times 2$ (Ages $\times$ Experience Levels $\times$ Rhythmic Conditions [R vs. I]) ANOVA. The only significant effects were those of experience, $F(2,71)$ $=10.55, p<.001, M S E=627,371.2, R^{2}=.142$, and rhythmic condition, $F(1,71)=110.63, p<.001, M S E=170,176.2, R^{2}$ $=.202$. Experienced listeners performed better with the slow thresholds, as shown in Table 2 (and as implied by the SF $\times$ Experience correlation in Table 1). Slow thresholds were 27\% slower with rhythmically intact melodies than with isochronous melodies, as shown in Table 3.

\section{Fast-Slow}

In our first FS analysis we did not include the FSG condition, to keep this analysis parallel to the overall analysis provided earlier. This gave a $3 \times 3 \times 2$ (Ages $\times$ Experience Levels $\times$ Rhythmic Conditions [R vs. I]) design. The only significant effects were those of age, $F(2,71)=4.44, p<.05, M S E=$ $4,186.7, R^{2}=.071$; experience, $F(2,71)=5.56, p<.01, M S E$ $=4,186.7, R^{2}=.089$; and rhythmic condition, $F(1,71)=$ 12.94, $p<.01, M S E=1,494.4, R^{2}=.044$. Older listeners had slower thresholds than younger, with thresholds of 163,187 , and $200 \mathrm{~ms} /$ note going from younger to older. Note that the drop in performance between the younger and the middle groups is about twice the drop between the middle and oldest groups ( $15 \%$ vs. $7 \%$ ). Experienced listeners performed better than inexperienced ones, as shown in Table 2. (These effects of age and experience directly parallel the correlations shown in Table 1.) Fast thresholds were about $10 \%$ slower with rhythmically intact melodies than with isochronous melodies, as shown in Table 3.

In the next analysis of FS data we included the presence or absence of a temporal gap between repetitions of the melody as a variable. This gave a design with $3 \times 3 \times 2 \times 2$ (Ages $\times$ Experience Levels $\times$ Rhythmic Conditions [R vs. I]) $\times$ Gap Conditions [FS vs. FSG]). As before, the effect of age was significant, $F(2,71)=4.40, p<.05, M S E=6,223.7, R^{2}=$ .052 ; as were the effects of experience, $F(2,71)=9.78, p<$ $.001, M S E=6,223.7, R^{2}=.115$; and rhythmic condition, $F(1$, $71)=29.76, p<.001), M S E=1,600.9, R^{2}=.045$. The effect of the gap approached significance, $F(1,71)=2.72, p<.10$, $M S E=1,759.4, R^{2}=.005$, with better performance with than without. Means pertaining to those effects are shown in Tables 2 and 3.

The results of the ANOVAs including all trials closely paralleled the results of the previous ANOVAs on just the correct trials, and all the means corresponding to those in Tables 2, 3, and 4 were within $2 \%$ of those values, except for three entries in the FSG condition that were within $6 \mathrm{~ms} /$ note of the means shown. There was one difference in the ANOVA results when all trials were included: In the ANOVA contrasting the FS and FSG conditions the beneficial effect of including the gap attained significance, $F(1,71)=8.40, p<.01, M S E=1,474.3$, $R^{2}=.013$.
Table 4

Recognition Thresholds and Standard Deviations in Milliseconds/Note for Isochronous Presentation in the FSG Condition

\begin{tabular}{lrrrr}
\hline & \multicolumn{4}{c}{ Experience } \\
\cline { 2 - 4 } Age & Low & Mid & Pro & $M$ \\
\hline Younger & & & & \\
$M$ & 155 & 178 & 123 & 152 \\
$S D$ & 30 & 54 & 37 & \\
Middle & & & & \\
$M$ & 180 & 173 & 141 & 165 \\
$S D$ & 52 & 50 & 40 & \\
Older & & & & \\
$M$ & 209 & 176 & 130 & 172 \\
$S D$ & 67 & 39 & 47 & \\
Overall $M$ & 181 & 176 & 131 & \\
\hline
\end{tabular}

Note. $\quad$ FSG $=$ fast-to-slow, gapped; Pro = professional.

\section{Errors}

We analyzed the proportion of identification errors in the six conditions defined by three speed-and-gap conditions (SF, FS, and FSG) and two rhythmic conditions ( $I$ and R): a $3 \times 3 \times$ $3 \times 2$ (Ages $\times$ Experience Levels $\times$ Speed-Gap Conditions $\times$ Rhythmic Conditions) design. The only significant effects were those of rhythmic condition, $F(1,71)=27.59, p<.001$, $M S E=1.14, R^{2}=.046$, and the Speed-and-Gap $\times$ Rhythmic Condition interaction, $F(2,71)=4.90, p<.01, M S E=0.74$, $R^{2}=.011$. Those results are shown in Table 5 . Rhythmic presentation led to fewer errors, especially on FS trials. Qualitatively, most errors involved confusions of highly similar songs, such as substituting "Mary Had a Little Lamb" for "London Bridge" on the basis of their similar rhythm and membership in the same category (nursery songs), and this was especially true in the isochronous condition. There were no other effectsin particular, there were no effects of age or experience on errors. (The mean proportions of errors for the age groups in ascending order were $.24, .20$, and .25 ; and for experience were $.26, .24$, and .19.)

In spite of the relatively large number of errors, we believe that this pattern merely indicates that listeners often tended to perform a recognition task rather than a strict identification task. Listeners were generally terminating trials as soon as they recognized a melody, and then naming the melody they recognized. Most often they gave it the correct name, but sometimes the name was wrong because of confusions between similar tunes, as noted previously. We do not believe that this biased the results concerning our main conclusions because (1) there were no effects due to age or experience in the analysis of errors, and (2) we obtained essentially the same ANOVA results when all trials were included as we did with only correct trials.

\section{Discussion}

\section{Aging and Expertise}

The relative effects of age and expertise on fast and slow melody identification can be seen clearly in Table 1. Expertise 
Table 5

Proportions of Errors and Standard Deviations for the SF, FS, and FSG Conditions and Rhythmic and Isochronous Presentation

\begin{tabular}{lllll}
\hline & \multicolumn{4}{c}{ Condition } \\
\cline { 2 - 4 } Presentation & SF & FS & FSG & $M$ \\
\hline Isochronous & & & & \\
$\quad M$ & .27 & .29 & .27 & .27 \\
$S D$ & .20 & .20 & .21 & \\
Rhythmic & .23 & .16 & .16 & .19 \\
$\quad M$ & .16 & .17 & .20 & \\
$S D$ & .25 & .23 & .22 & .23 \\
Overall $M$ & &
\end{tabular}

Note. $\quad$ SF $=$ slow-to-fast; FS = fast-to-slow; FSG $=$ fast-to-slow, gapped.

accounts for more than $20 \%$ of the variation of both FS and SF performance, whereas age accounts for only $7 \%$ of the variation of FS performance and appears unrelated to SF performance. The relative importance of expertise is all the more striking because, although the correlations with age used the finely coded variable "age in years," expertise was necessarily more coarsely coded (in this case in four categories, leading to a less powerful test than that for age). The relative importance of expertise versus age converges with the results of Charness's (1985) studies of accuracy in bridge bidding and chess. It also converges with the results of Halpern, Bartlett, and Dowling (1995), who in four experiments on recognition of transpositions of novel melodies found a preponderance of experience over age effects. In two experiments Halpern et al. found the effects of age and experience to be about equal (omega-squared around .10 ), and in the other two experiments found experience effects to be more than four times as large as age effects (omegasquared around $.35 \mathrm{vs.} .01-.08$ ). Halpern et al. have speculated that the greater impact of experience effects occurs especially in those tasks where high-level musical encoding of the stimuli is required (for example, where interference is introduced into the task).

The effects of age on speeded processing are spread rather evenly across the 60-year range we studied; there does not appear to be an accelerated slowing during the later years in these data. And given the correlation of less than .3 between age and FS thresholds there is considerable overlap in the distributions of the oldest and youngest listeners; for example, $25 \%$ of listeners over 65 performed better than the median for listeners under 25 . The spread of this effect across the life span closely resembles the pattern found by Schaie (1989) with paper-and-pencil tests for speed of processing. And we should add the caution suggested by Schaie's longitudinal data, that a linear relationship across the life span for cross-sectional data does not mean that each individual experiences a gradual decline in performance with increasing age. Rather, individual performance is very likely quite stable over relatively long periods.

We believe that the more expert listeners' better performance with both fast and slow stimuli can be attributed to their having developed better strategies for pattern recognition over years of perceptual learning with melodic stimuli over a wide range of tempos. In particular, we can reject the possibility that the experts performed better because of greater familiarity with the specific materials used in this experiment, because identification errors did not vary systematically with experience. This lack of effect of age and expertise on errors also suggests that the task and melodies we used were of an appropriate level of difficulty for all listeners. The use of a small, closed set of highly familiar melodies helped make the task roughly equivalent for both experienced and inexperienced listeners. Increased familiarity with the specific materials being used was apparently not a factor in the experienced listeners' superior performance.

\section{Encoding and Age}

In fact, the pattern of data in Table 1 points to encoding problems as the source of difficulty with increasing age. In particular, the data show neither the slowing of thresholds for both FS and SF trials, as predicted by the slowed-clock model, nor the slowing of FS thresholds and speeding up of SF thresholds predicted by the increased-overhead and information-loss models (as applied to memory in general). The lack of an effect of age on SF performance is not due to insensitivity of the task. because expertise had equally strong effects on both SF and FS performance.

The fact that age affected only FS performance suggests that the generalized slowing observed with increasing age is concerned with stimulus encoding processes. This is because encoding processes are the component involved in FS performance but not SF performance. This conclusion converges with Salthouse's (1996a, 1996b) conclusions from his survey of the literature on cognitive slowing with age. Salthouse, reviewing evidence that very little in the way of age-related effects can be attributed to tasks providing relatively long stimulus durations, notes that for several types of task early stimulus encoding processes need to be taken into account as a source of cognitive slowing. What the present results may mean is that whereas rapid stimulus encoding declines with age across the life span, the domainspecific skill of constructing the representation of an abnormally slow tune from fragments remains intact.

\section{Rhythmic Versus Isochronous Presentation}

The interaction shown in Table 3 indicates that rhythmic presentation aided melody identification for slow melodies, whereas isochronous presentation provided an advantage for fast melodies. This pattern held equally for the melodies that appeared in both the I and $\mathrm{R}$ conditions and the melodies that appeared in only one of those conditions. This interaction suggests that listeners rely on different types of information and employ different strategies in the FS and SF tasks. On FS trials what is most important is the efficient transmission of pitch and pitchcontour information at a rapid rate. The very short duration notes that occasionally result from presentation with the rhythmic pattern of the intact tunes tend to get lost in the stream of notes. Thus isochronous presentation results in faster thresholds on FS trials. Isochronous presentation, however, carries a cost in increased ambiguity, reflected in higher error rates on FS-I trials than FS-R trials. On SF trials, in contrast, rhythmic 
presentation improves thresholds and reduces errors slightly because at the slower tempo listeners are able to focus more on the individual notes and the rhythmic cues to the identity of the melody.

The differences in strategy on FS and SF trials are related to a point that Warren et al. (1991) have made regarding the FS stimuli: When the tempo crosses the recognition threshold the melody forms a coherent gestalt; it appears effortlessly in working memory as a meaningful whole. Recognition is an automatic, "data driven" process. With SF stimuli, in contrast, recognition at the slow thresholds observed here requires active construction of the pattern representation by the listener. The slowly occurring notes must be held, one by one, long enough for the listener to construct an image of the tune in working memory.

\section{Gapped Presentation}

We expected adding the gap between iterations of the FS stimuli to aid in parsing the melody by indicating its start, and hence to produce better performance, especially with the older listeners. The gap did help, but about equally across all age groups and levels of experience. Though gap did not interact with the other variables, it nevertheless led to the consistently fastest thresholds in the whole experiment in the FSG-I condition. Those data are shown in Table 4. Though the interaction is not statistically significant, it is clear that the professionals performed about equally well no matter what their age. In fact, only for the inexperienced listeners is there a noticeable effect of age. This suggests that musicians with at least moderate training are able to make use of the gap in solving the parsing problem for rapid recycled isochronous melodies, and that this ability can be used to compensate for whatever difficulties are encountered in this task with increasing age. (Several older professionals commented that they would have liked to have had some signal indicating the start of the tune in the SF condition. They found the SF-I condition without such a marker quite confusing, because they were analyzing the contribution of each note separately as it occurred.) However, further research focused on this issue is necessary to demonstrate a statistically significant effect of the gap in facilitating parsing for musically experienced listeners.

\section{General Observations}

The present melody-identification thresholds are in the same ballpark as those of Warren et al. (1991), who found overall median thresholds of 160 and $1,280 \mathrm{~ms} /$ note, but medians as fast as $113 \mathrm{~ms} /$ note and as slow as $2,180 \mathrm{~ms} /$ note for particular melodies. Both the present results and those of Warren et al. represent a wider range than those found by Halpern (1988) for fastest and slowest tempos of imagined familiar songs (366 and $923 \mathrm{~ms}$ (beat). There are several possible sources for this difference inherent in the differences between the tasks. Halpern's scale of possible responses was limited ( 288 to $1,500 \mathrm{~ms} /$ beat-well within the range of Warren et al.'s responses). We simply do not know whether Halpern's listeners were unable to imagine the songs going as fast or as slow as their identification thresholds, or whether they were limited by the range of the response scale.
The fact that the slow thresholds are slower and the fast thresholds faster than in the Warren et al. (1991) study is probably due to the fact that the present listeners appear uniformly to have been treating the experiment more as a recognition task than as an identification task, as noted previously in connection with the error rates. Listeners in general were terminating trials as soon as they recognized the melody, but sometimes gave it the wrong name. Recognition requires less stimulus information than identification, and in basing their responses on a recognition criterion listeners were probably able to respond sooner. Maylor's (1991) data suggest that older listeners may have more difficulty with identifying melodies than recognizing them. It is possible that if we had insisted on a stricter identification task greater age differences might have appeared, though it is important to note that numbers of errors were related to neither age nor expertise.

Finally, we wish to call attention to the methodological importance not only of including a wide range of ages and levels of expertise in this study, but also of ensuring that intermediate levels of both age and expertise were included as well as the extremes. Not only does this approach provide for a fair comparison of the relative importance of age and expertise, but also the steady and gradual effect of age on the FS task would have escaped our attention had we included only younger and older groups. In fact, further research might well focus on changes in perceptual encoding during the middle years.

\section{References}

Cerella, J. ( 1990). Aging and information-processing rate. In J. E. Birren \& K. W. Schaie (Eds.), Handbook of psychology of aging (3rd ed., pp. 201-221). Orlando, FL: Academic Press.

Charness, N. (1985). Aging and problem-solving performance. In N. Charness (Ed.), Aging and human performance (pp. 225-259). New York: Wiley.

Dowling, W. J. ( 1973). The perception of interleaved melodies. Cognitive Psychology, 5, 322-337.

Dowling, W. J. (1986). Context effects on melody recognition: Scalestep versus interval representations. Music Perception, 3, 281-296.

Dowling. W. J. (1992). Perceptual grouping, attention and expectancy in listening to music. In J. Sundberg (Ed.), Gluing tones: Grouping in music composition, performance and listening (No. 72, pp. 7798). Stockholm: Royal Swedish Academy of Music.

Dowling, W. J., \& Harwood, D. L. (1986). Music cognition. Orlando, FL: Academic Press.

Halpern, A. R. (1988). Perceived and imagined tempos of familiar songs. Music Perception, 6, 193-202.

Halpern, A. R., Bartlett, J. C., \& Dowling, W. J. (1995). Aging and experience in the recognition of musical transpositions. Psychology and Aging, 10, 325-342.

Hendershott, G. (1990). Cakewalk (Version 4. 0) [Computer software]. Watertown, MA: Twelve Tone Systems.

Krampe, R. T., \& Ericsson, K. A. (1996). Maintaining excellence: Deliberate practice and elite performance in young and older pianists. Journal of Experimental Psychology: General, 125, 331-359.

Maylor, E. A. (1991). Recognizing and naming tunes: Memory impairment in the elderly. Journals of Gerontology: Psychological Sciences, 46, $\mathrm{P} 207-\mathrm{P} 217$.

Myerson, J., Hale, S., Wagstaff, D., Poon, L. W., \& Smith, G. A. (1990). The information-loss model: A mathematical theory of age-related cognitive slowing. Psychological Review, 97, 475-487.

Salthouse, T. A. (1984). Effects of age and skill in typing. Journal of Experimental Psychology: General, 113, 345-371. 
Salthouse, T. A. (1996a). Constraints on theories of cognitive aging. Psychonomic Bulletin and Review, 3, 287-299.

Salthouse, T. A. (1996b). General and specific speed mediation of adult age differences in memory. Journals of Gerontology: Psychological Sciences, 51B, P30-P42.

Schaie, K. W. (1989). Perceptual speed in adulthood: Cross-sectional and longitudinal studies. Psychology and Aging, 4, 443-453.

Trainor, L. J., \& Trehub, S.E. (1989). Aging and auditory temporal sequencing: Ordering the elements of repeating tone patterns. Perception \& Psychophysics, 45, 417-426.

Warren, R. M., \& Bashford, J. A., Jr. (1993). When acoustic sequences are not perceptual sequences: The global perception of auditory patterns. Penception \& Psychophysics, 54, 121-126.

Warren, R. M., Gardner, D. A., Brubaker, B. S., \& Bashford, J. A., Jr. (1989, October). Melodic and nonmelodic pitch patterns: Effects of duration on perception. Paper presented at the meeting of the First International Conference on Music Perception and Cognition, Kyoto, Japan.

Warren, R. M., Gardner, D. A., Brubaker, B. S., \& Bashford, J. A., Jr. (1991). Melodic and nonmelodic sequences of tones: Effects of duration on perception. Music Perception, 8, 277-290.

Weber, C. M. von. (1989). Der Freischuitz. [The Freeshooter; Recorded by Elisabeth Grümmer, Rita Streich, Wilhelm Furtwängler, \& the Vienna Philharmonic Orchestra]. On Der Freischütz [Record]. Milan: Metromusica. (1954)

Wechsler, D. (1981). Wechsler Adult Intelligence Scale-Revised. New York: Psychological Corporation.

Received August 19, 1997

Revision received March 10, 1998

Accepted March 10, 1998 